\title{
EXPERIMENTAL AND NUMERICAL INVESTIGATION OF BASE ISOLATED SDOF SYSTEM IMPACT AGAINST BUMPERS UNDER HARMONIC BASE EXCITATION
}

\author{
Giulia Stefani ${ }^{1}$, Maurizio De Angelis ${ }^{1}$, and Ugo Andreaus ${ }^{1}$ \\ ${ }^{1}$ Department of Structural and Geotechnical Engineering, Sapienza University of Rome \\ Via Eudossiana 18, 00184 Rome, Italy \\ \{giulia.stefani,maurizio.deangelis,ugo.andreaus\}@uniroma1.it
}

\begin{abstract}
During strong earthquakes, base isolated systems (buildings, bridges, strategic facilities, equipment etc.) can impact against the surrounding moat wall because of the deformation of the isolator if the available separation distance is limited. A possible mitigation measure is the interposition of shock-absorbers (bumpers). In this work some of the results of an experimental laboratory campaign are presented. The experimental tests were carried out to investigate the dynamic response of a base-isolated single-degree-of-freedom (SDOF) oscillator, excited by a harmonic base acceleration and symmetrically constrained by two unilateral deformable and dissipative bumpers. Three different peak values of table acceleration (A), four amplitudes of the total gap between mass and bumpers $(G)$ and four types of bumpers $(B)$ were considered. In this work the attention is focused on some characteristics of the dynamics with impact, such as force and time of contact between mass and bumpers, coefficient of restitution and energy dissipated by the bumpers during the impact. The results of the experimental tests were used to identify the parameters of stiffness and damping of a numerical model able to simulate the behavior of the system by using a general-purpose computer code.
\end{abstract}

Keywords: Shaking table test, Numerical model, Base-isolated SDOF, Bumpers, Base excitation, Impact parameters. 


\section{INTRODUCTION}

Base-isolated structures can undergo large displacements when subjected to severe earthquakes, because of the lengthening of their fundamental periods induced by seismic isolation $[1,2]$. These large displacements, confined at the isolation system, can damage the isolators themselves or can lead to poundings with the surrounding moat wall or adjacent structures, if the width of the available seismic gap is not sufficient. The problem of pounding doesn't concern only structures, but also bridges and equipment $[3,4]$. The consequences of such pounding, that can range from damage of acceleration-sensitive equipment housed in the structure to severe structural damage, can be mitigated by reducing the impact stiffness. This can be done, for example, by introducing shock absorbers (bumpers), made of deformable and dissipative material, between the structure and the obstacle.

The dynamic response of a nonlinear base-isolated single-degree-of-freedom (SDOF) oscillator subjected to a harmonic base acceleration and constrained by two symmetrically arranged unilateral bumpers has been numerically investigated by Andreaus and De Angelis in [5], where possible scenarios of the system's response have been highlighted. Subsequently, the experimental response of a physical model of the SDOF has been studied in [6,7].

In this work, some of the results of an experimental laboratory campaign of tests, concerning the study of the SDOF oscillator, are presented. In particular, the evaluation of the impact parameters (time and force of contact, coefficient of restitution and energy dissipated by the bumpers during the impact) was made on the basis of the experimental data (times and velocities immediately before and after contact). Three different peak values of table acceleration Ai $(i=1,2,3)$, four amplitudes of the total gap between mass and bumpers $G j(j=1,2,3,4)$ and four types of bumpers $\mathrm{Bk}(\mathrm{k}=1,2,3,4)$ were considered.

The results of the experimental tests were also used to identify the parameters of a numerical model able to simulate the behavior of the system by using a general-purpose computer code.

The paper is organized as follows: the experimental setup is described in Sect. 2; the impact parameters, calculated from the experimental data, are reported in Sect. 3; a numerical model able to simulate the behavior of the system is presented in Sect. 4; the main conclusions are drawn in Sect. 5.

\section{EXPERIMENTAL SETUP}

The physical model of the SDOF system consists of a rigid body, composed of six plates of mild steel jointed through bolts, that can be treated as a lumped mass $M$, an elastomeric isolator (damper) and a couple of elastomeric shock absorbers (bumpers) symmetrically installed on steel stands which are bolted onto the base plate. The damper is bolted to the lower plate of the mass in a central position. The mass is supported by four spherical bearings, rotating within unidirectional guides (Figure 1).

The experimental campaign was performed using a shaking table, and the measured parameters were absolute accelerations and displacements of the table and of the mass.

Four different values of the distance between bumpers and mass (total gap, defined as the sum of left and right gaps), obtained by adjusting the screws at the fronts of the stands, were considered $(\mathrm{G} 1=15 \mathrm{~mm}, \mathrm{G} 2=20 \mathrm{~mm}, \mathrm{G} 3=25 \mathrm{~mm}, \mathrm{G} 4=30 \mathrm{~mm})$. 


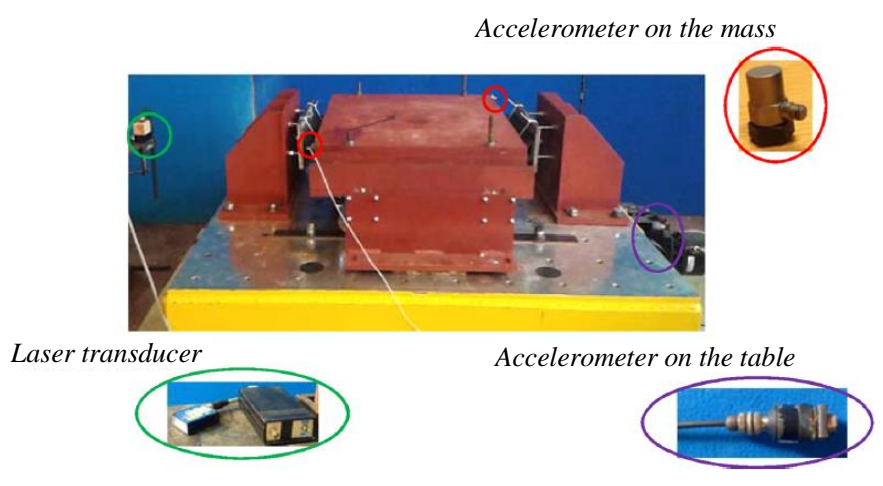

a)

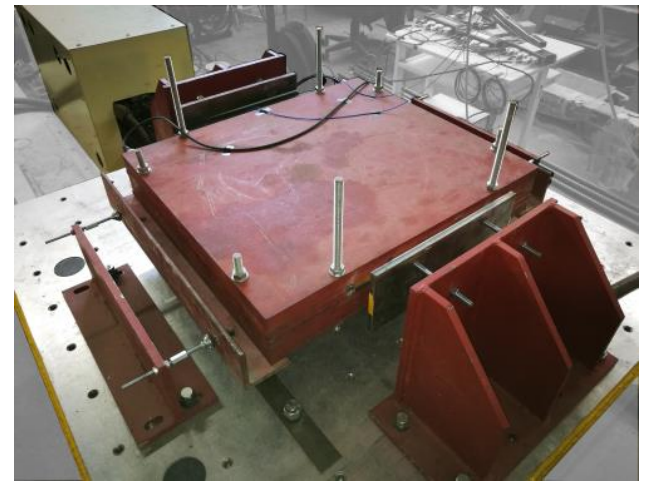

b)

Figure 1: Experimental setup: a) side view and sensors; b) perspective view.

Four different types of bumpers were employed (B1, B2, B3, B4), with different contact lengths and obtained from three types of cross section (Figure 2).
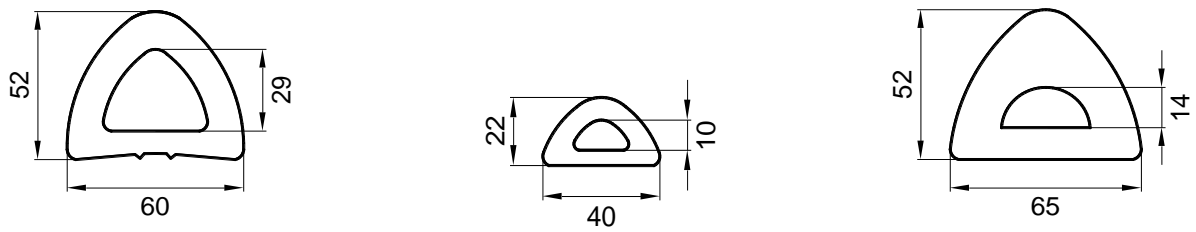

Figure 2: Cross sections of bumpers: a) B1 and B2: MB 60x52 mm, B1: L = $100 \mathrm{~mm}, \mathrm{~B} 2: \mathrm{L}=400 \mathrm{~mm}$; b) B3: MB 40x22 mm, L= $400 \mathrm{~mm}$; c) B4: AP 65x52 mm, L= $400 \mathrm{~mm}$.

The system was excited by a step-wise forward and backward Sine Sweep signal in displacement control, in the frequency range $f=0.5-5.0 \mathrm{~Hz}$ with step $\Delta f=0.1 \mathrm{~Hz}$, in order to impose three different values of peak table acceleration $(\mathrm{A} 1=0.03 \mathrm{~g}, \mathrm{~A} 2=0.04 \mathrm{~g}, \mathrm{~A} 3=0.05$ $\mathrm{g}$, where $\mathrm{g}$ is the gravity's acceleration), with a sufficient number of cycles to reach steady state.

\section{EXPERIMENTAL EVALUATION OF IMPACT PARAMETERS}

Among the various aspects investigated, in this work the attention is focused on the results obtained in the presence of bumpers which were used to identify some characteristic parameters of the dynamics with impact, such as force and time of contact between mass and bumpers, coefficient of restitution and energy dissipated by the bumpers during the impact. These parameters were evaluated from the experimental data, considering the steady-state in correspondence to the resonance frequency. In case of rigid body impact, the contact time is very small, and the contact force prevails over other involved forces [8].

By analyzing the time histories of absolute acceleration in steady-state resonance condition (Figure 3), it was possible to identify the times of beginning and end of contact (respectively $t_{\mathrm{i}}$ and $t_{\mathrm{o}}$ ), and thus calculate the duration of the contact interval:

$$
\Delta t_{\mathrm{c}}=t_{\mathrm{o}}-t_{\mathrm{i}}
$$




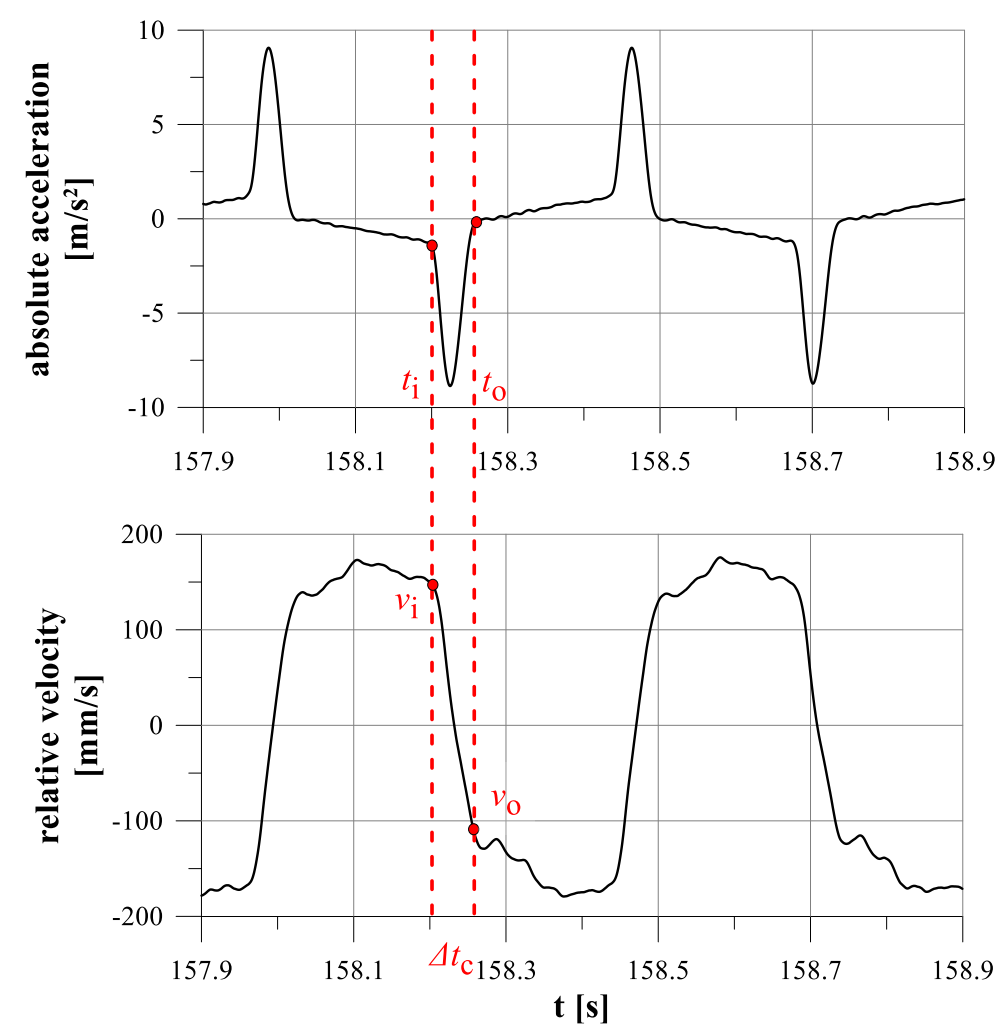

Figure 3: Time histories of absolute acceleration and relative velocity in steady-state resonance condition (B4G4-A3).

The coefficient of restitution $s$ describes the loss of kinetic energy due to wave propagation and deformation of the contact area caused by the impact. It is defined as the ratio between the relative velocities immediately after, $v_{\mathrm{o}}$, and immediately before, $v_{\mathrm{i}}$, the contact (Figure 3):

$$
s=-v_{\mathrm{o}} / v_{\mathrm{i}}
$$

It depends on material, geometry of impact bodies and relative velocities of the colliding solids $[9,10]$. The coefficient, so defined, varies within the range $s \in[0,1]$. The limit values correspond to perfectly inelastic and perfectly elastic impact, respectively.

Using the impulse momentum law, with reference to the time interval $\Delta t_{\mathrm{c}}$, the mean contact force, acting on the mass, con be evaluated as follows:

$$
F_{\mathrm{c}}=I / \Delta t_{\mathrm{c}}
$$

where $I$ is the change in momentum during impact:

$$
I=M(1+s) v_{\mathrm{i}}
$$

Finally, the energy dissipated during the impact, equal to the difference between the kinetic energies of the mass $M$ immediately before $\left(E_{\mathrm{ki}}\right)$ and immediately after $\left(E_{\mathrm{ko}}\right)$ the contact with the bumper, can be calculated from the coefficient of restitution through the following expression [11]:

$$
E_{\mathrm{d}}=\left(1-s^{2}\right) E_{\mathrm{ki}}
$$

In the following figures, the trends of the impact parameters are depicted as a function of the peak table acceleration A (Figure 4), the amplitude of the total gap G (Figure 5) and the stiffness of the bumper (Figure 6). Missing values correspond to combination of the parameters A-G-B for which the impact has not occurred. 
To each bumper B corresponds a color (red for B1, blue for B2, black for B3 and green for B4); to each value of total gap G corresponds a marker (square for G1, diamond for G2, triangle for G3 and circle for G4); to each value of peak table acceleration A corresponds a line style (dotted for A1, dashed for A2 and solid for A3). From Figure 4 it can be observed that all the impact parameters increase with the peak table acceleration A. While bumpers B2 and B3, and often also B4, behave in a similar way, bumper B1 exhibits quite different trends. As concerns the coefficient of restitution $s$, from Figure 4 a it can be observed that it always assumes values lower than 1 . The lowest values are those associated with the bumper B1, whereas the curves corresponding to the other three bumpers are very close to each other, especially for large values of A. Consequently, it is expected that, for those three bumpers, the influence of the total gap $\mathrm{G}$ on $s$ decreases with increasing of A.

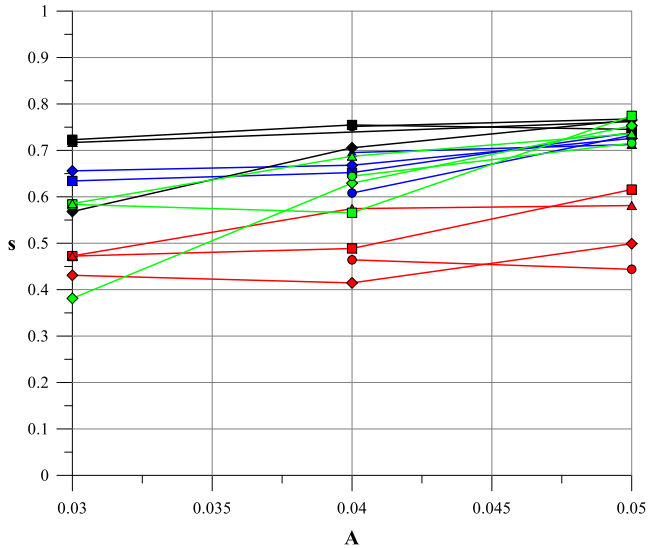

a)

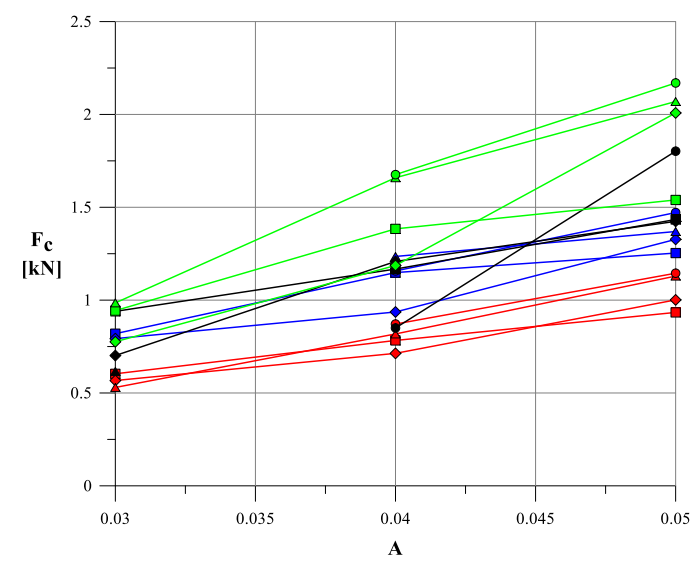

c)

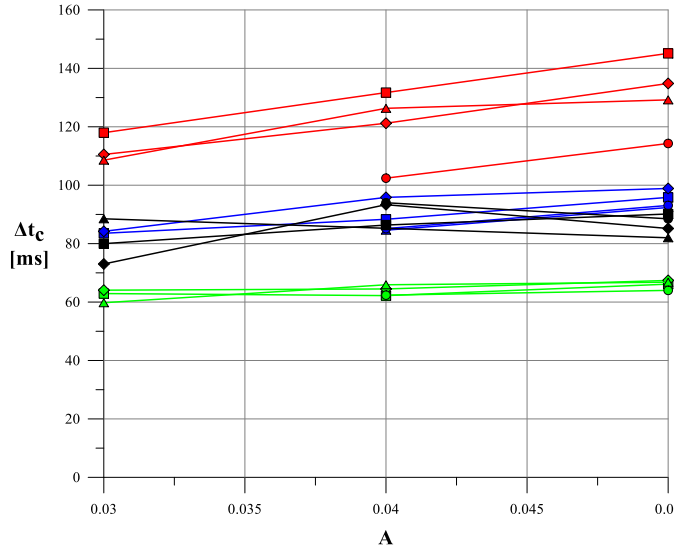

b)

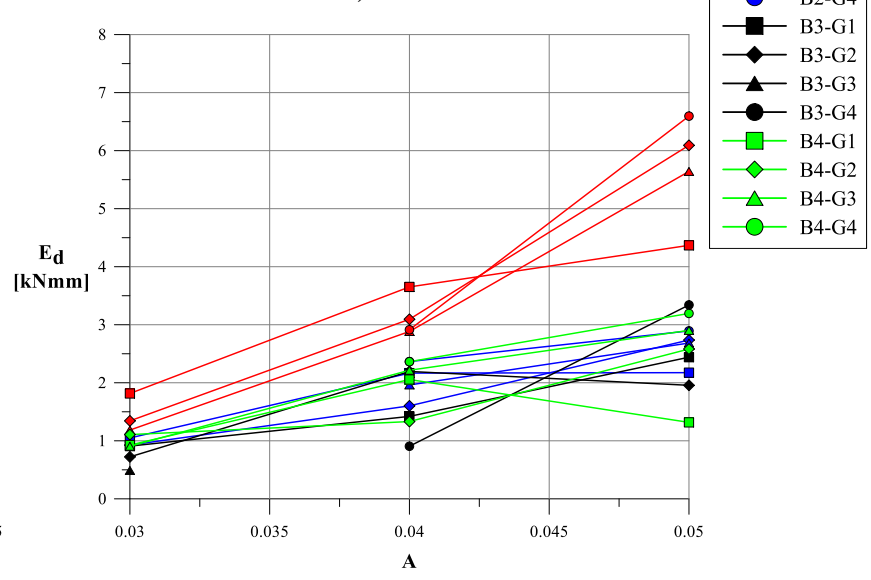

d)

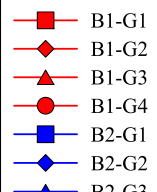

$\longrightarrow$ B2-G2 B2-G4 $\checkmark$ B3-G2 B3-G4 - B4-G1 B4-G3 B4-G4

Figure 4: Impact parameters vs. A with the variation of B and G: a) coefficient of restitution; b) contact time; c) contact force; d) dissipated energy.

As concerns the contact time $\Delta t_{\mathrm{c}}$ (Figure $4 \mathrm{~b}$ ), it is very short, of the order of milliseconds. The increase of $\Delta t_{\mathrm{c}}$ with $\mathrm{A}$ is due to the fact that the higher $\mathrm{A}$, the greater the penetration of the mass in the bumpers. Furthermore, in the case of bumper B4, it is worth noting that not only the contact time does not vary significantly with $A$, but also $G$ does not influence too much the duration of contact because the curves, corresponding to the four values of $\mathrm{G}$, are very close to each other. As the stiffness of the bumper decreases, the influence of A increases, and it is more evident the difference in $\Delta t_{\mathrm{c}}$ when varying $\mathrm{G}$, especially for bumper B1. Bumpers B2 and B3 exhibit values of $\Delta t_{\mathrm{c}}$ very close to each other. 
As concerns the mean contact force $F_{\mathrm{c}}$, from Figure $4 \mathrm{c}$ it can be observed that it increases almost linearly with $A$. The lower values are those associated with the more deformable bumper B1, whereas the larger values are those corresponding to the stiffer bumper B4. B2 and B3 are placed in an intermediate position. As concerns the energy dissipated during impact $E_{\mathrm{d}}$, from Figure $4 \mathrm{~d}$ it can be observed that, except for some cases, it increases with A. The values of $E_{\mathrm{d}}$ associated with the bumper B1 are always larger and differ significantly from the other bumpers, especially for large values of A. The curves corresponding to the other bumpers are very close to each other.

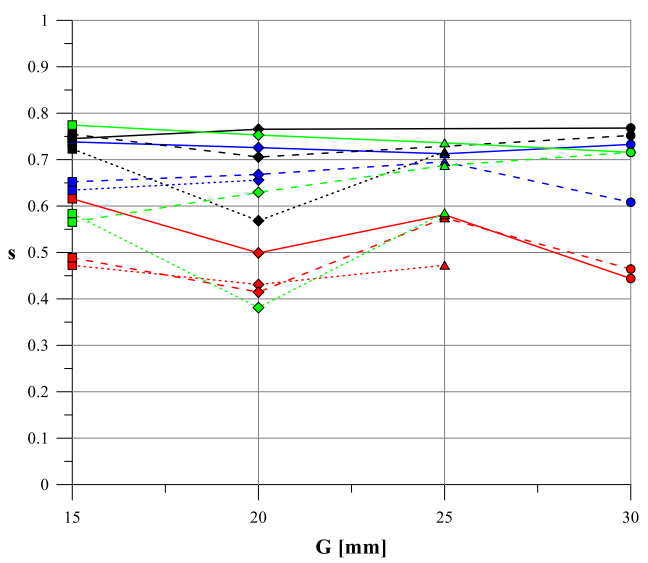

a)

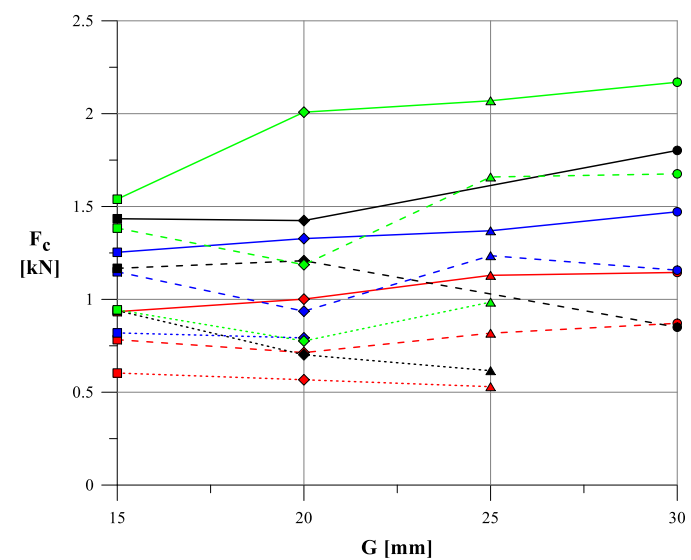

c)

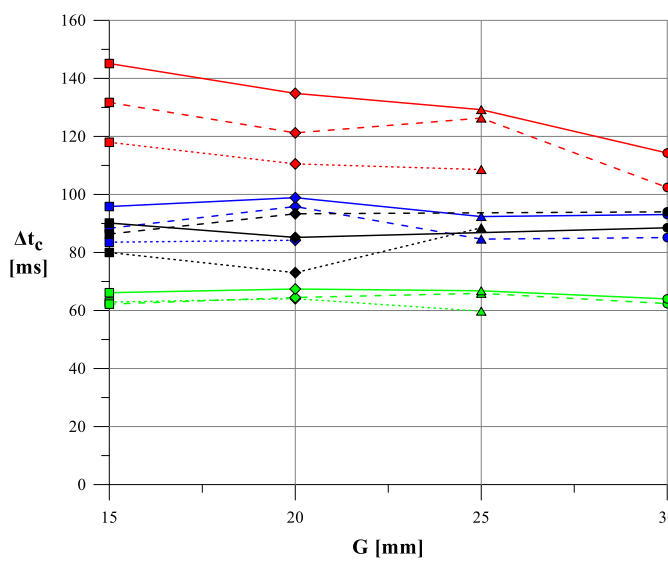

b)

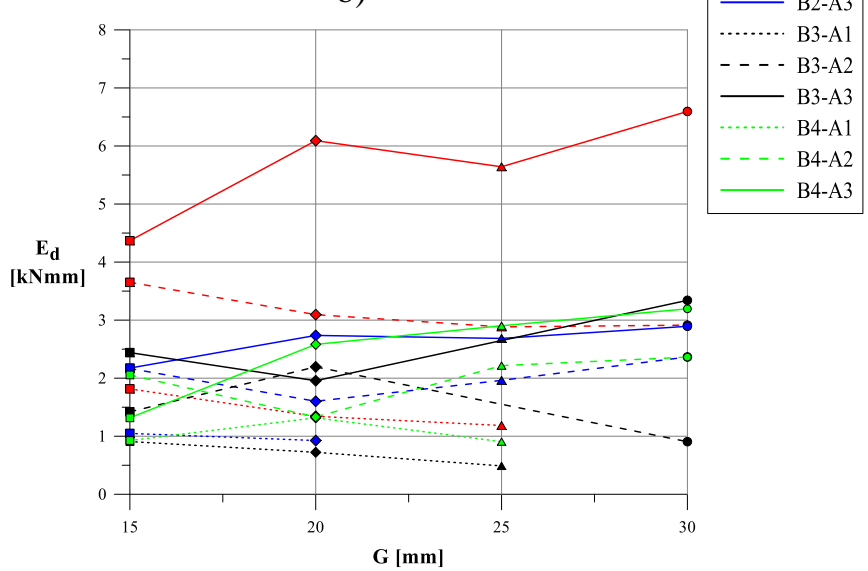

d)

Figure 5: Impact parameters $v s . \mathrm{G}$ with the variation of B and A: a) coefficient of restitution; b) contact time; c) contact force; d) dissipated energy.

From Figure 5 it can be observed that also the amplitude of the total gap $\mathrm{G}$ influences the impact parameters. The coefficient of restitution (Figure 5a) does not vary significantly with $\mathrm{G}$, especially for large values of $\mathrm{A}$. As concerns $\Delta t_{\mathrm{c}}$ (Figure $5 \mathrm{~b}$ ), it is not significantly influenced by $\mathrm{G}$ for bumpers B2, B3 and B4, whereas it decreases with $\mathrm{G}$ for bumper B1. The curves associated with bumper B4 are very close to each other, indicating a small influence of A. This influence increases as the stiffness of the bumper decreases. The mean contact force $F_{\mathrm{c}}$ (Figure $5 \mathrm{c}$ ) does not vary significantly or increases slightly with $\mathrm{G}$. The higher values are associated with the pair B4-A3. The energy dissipated (Figure 5d), attains value in the range 0.5-3.5 kN mm for all values of G. The curve corresponding to the pair B1-A3 distances itself significantly from the others and it is associated with the larger values of $E_{\mathrm{d}}$. The same pair of parameters corresponds to the greater value of $\Delta t_{\mathrm{c}}$. 


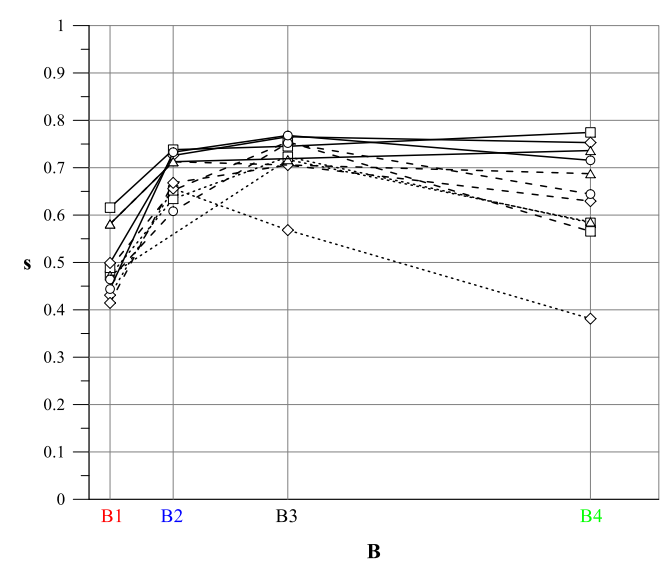

a)

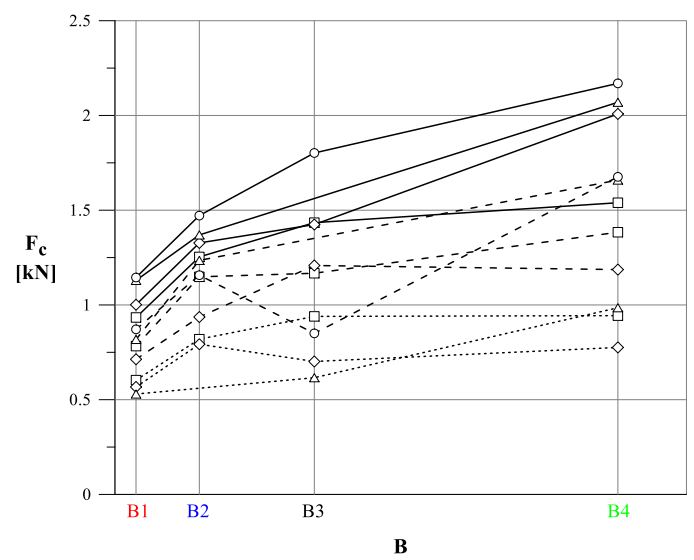

c)

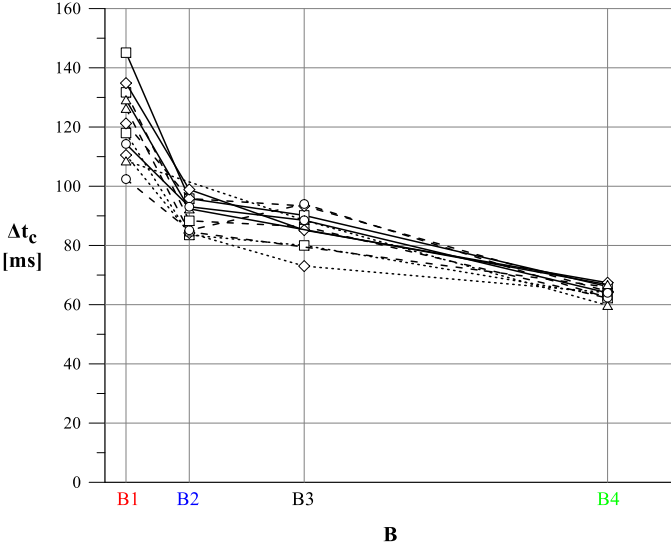

b)

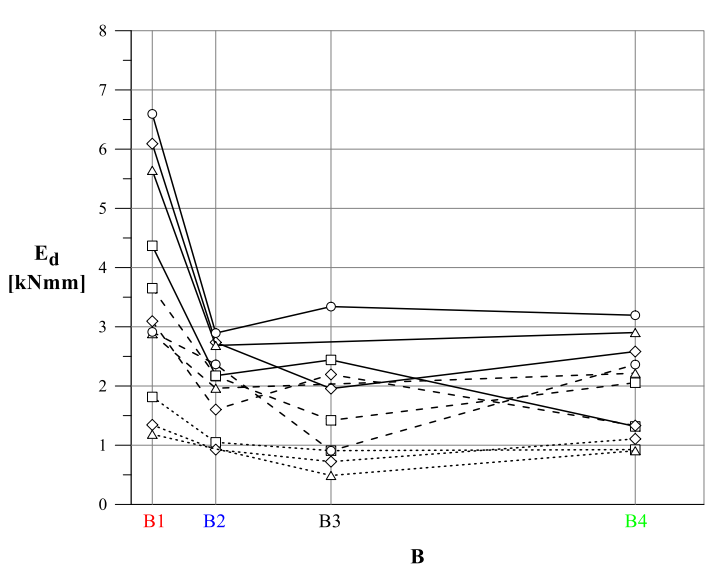

d)

Figure 6: Impact parameters vs. B with the variation of $\mathrm{G}$ and $\mathrm{A}$ : a) coefficient of restitution; b) contact time; c) contact force; d) dissipated energy.

As concerns the influence of the stiffness of the bumper B, from Figure 6a it can be observed that, as it increases, the coefficient of restitution first increases and then it stands at a constant value or decreases. The reduction increases as A decreases. Only the curve corresponding to the pair G2-A1 gradually diverges more and more from the others as B increases. Passing from B1 to B4, that is increasing the stiffness of the bumper, the penetration of the mass into the bumper decreases. Consequently, $\Delta t_{\mathrm{c}}$ decreases (Figure $6 \mathrm{~b}$ ) because the mass remains in contact with the bumper for a shorter time. It is worth noting that all the curves are gradually closer to each other as $\mathrm{B}$ increases. The mean contact force $F_{\mathrm{c}}$ increases with the stiffness of the bumper (Figure 6c) and the larger values are associated with the higher values of peak table acceleration. As concerns the energy dissipated during the impact (Figure 6d), it is not significantly influenced by $\mathrm{B}$ for small values of $\mathrm{A}$. With increasing of $\mathrm{A}, E_{\mathrm{d}}$ first decreases with $\mathrm{B}$, with a reduction that is all the more significant the greater is $\mathrm{A}$, then stands at a constant value or does not vary significantly. Finally, as previously observed, also from it can be noticed that bumpers B2 and B3 correspond more or less the same values of the parameters 


\section{NUMERICAL MODEL}

The results of the experimental tests were used to identify the parameters of stiffness and damping of a numerical model able to simulate the behavior of the SDOF system, by using a general-purpose computer code (Sap2000 v.20). The model, depicted in Figure 7a, consists of a mass $M$, a nonlinear isolation damper, in the following text denoted by the subscript " $\mathrm{d}$ ", and two bumpers, in the following text denoted by the subscript " $b$ ".

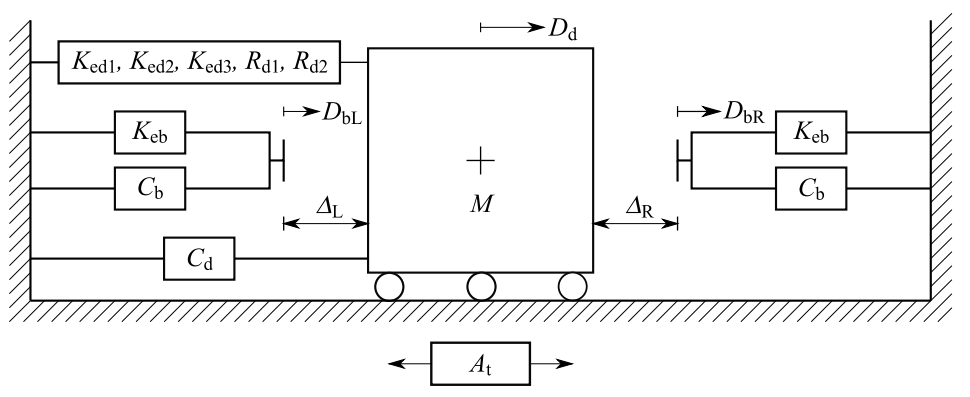

a)

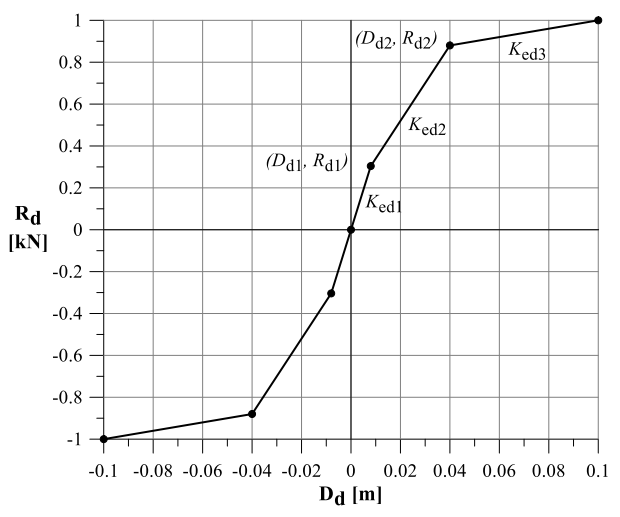

b)

Figure 7: a) Numerical model; b) Trilinear constitutive law of damper.

$D_{\mathrm{d}}$ and $D_{\mathrm{bj}}(j=R, L)$ denote the relative displacement of mass, right and left bumper respectively with respect to the table; in the following equations the superimposed dot denotes derivation with respect to time. The gap, that is the distance between the mass $M$ and the single bumper, is denoted by $\Delta_{\mathrm{j}}(t)(\mathrm{j}=R, L)$. The system is subjected to a harmonic base acceleration $A_{\mathrm{t}}(\mathrm{t})=A_{\mathrm{G}} \sin (\Omega t)$.

The experimental and numerical results where compared considering the combination of investigated parameters B4-G4-A3. The piece-wise linear elastic behavior of the damper [12] (Figure $7 \mathrm{~b}$ ) was modeled with stiffness $K_{\mathrm{ed} 1}=38 \mathrm{kN} / \mathrm{m}$ between $D_{\mathrm{d}}=0$ and $D_{\mathrm{d}}=D_{\mathrm{d} 1}=8 \mathrm{~mm}$, $K_{\text {ed } 2}=18 \mathrm{kN} / \mathrm{m}$ between $D_{\mathrm{d}}=D_{\mathrm{d} 1}=8 \mathrm{~mm}$ and $D_{\mathrm{d}}=D_{\mathrm{d} 2}=40 \mathrm{~mm}, K_{\mathrm{ed} 3}=2 \mathrm{kN} / \mathrm{m}$ for $D_{\mathrm{d}}>D_{\mathrm{d} 2}$ $=40 \mathrm{~mm}$; the linear viscous behavior of damper was identified by the damping coefficient $C_{\mathrm{d}}$ $=1.1 \mathrm{kNs} / \mathrm{m}$. The linear elastic behavior of the bumper was modeled with a stiffness $K_{\mathrm{eb}}=$ $2200 \mathrm{kN} / \mathrm{m}$; the linear viscous behavior of the bumper was identified by the damping coefficient $C_{\mathrm{b}}=5 \mathrm{kNs} / \mathrm{m}$.

The dynamic response of the system is governed by the following equations of motion. Three situations exist, when the mass is oscillating, and they can be described as follows:

- the mass is not in contact with any of the bumpers

$$
\left\{\begin{array}{l}
M \ddot{D}_{\mathrm{d}}+C_{\mathrm{d}} \dot{D}_{\mathrm{d}}+R_{\mathrm{d}}=-M A_{\mathrm{G}} \sin (\Omega t) \\
C_{\mathrm{bj}} \dot{D}_{\mathrm{bj}}+K_{\mathrm{eb}} D_{\mathrm{bj}}=0
\end{array}\right.
$$

- the mass is in contact with the right bumper (RB)

$$
\left\{\begin{array}{l}
M \ddot{D}_{\mathrm{d}}+C_{\mathrm{d}} \dot{D}_{\mathrm{d}}+R_{\mathrm{d}}+C_{\mathrm{bR}} \dot{D}_{\mathrm{bR}}+K_{\mathrm{eb}} D_{\mathrm{bR}}=-M A_{\mathrm{G}} \sin (\Omega t) \\
C_{\mathrm{bL}} \dot{D}_{\mathrm{bL}}+K_{\mathrm{eb}} D_{\mathrm{bL}}=0
\end{array}\right.
$$


- the mass is in contact with the left bumper (LB)

$$
\left\{\begin{array}{l}
M \ddot{D}_{\mathrm{d}}+C_{\mathrm{d}} \dot{D}_{\mathrm{d}}+R_{\mathrm{d}}+C_{\mathrm{bL}} \dot{D}_{\mathrm{bL}}+K_{\mathrm{eb}} D_{\mathrm{bL}}=-M A_{\mathrm{G}} \sin (\Omega t) \\
C_{\mathrm{bR}} \dot{D}_{\mathrm{bR}}+K_{\mathrm{eb}} D_{\mathrm{bR}}=0
\end{array}\right.
$$

The definition of $R_{\mathrm{d}}$ appearing in Eqs. (6) above will be given in the Eqs. (7) below.

The piecewise-linear restoring force of the damper obeys the following constitutive law in the $\left(D_{\mathrm{d}} \geq 0, R_{\mathrm{d}} \geq 0\right)$-region (Figure $\left.7 \mathrm{~b}\right)$ :

$$
\begin{array}{lrl}
R_{\mathrm{d}}=K_{\mathrm{ed} 1} D_{\mathrm{d}} & 0 \leq R_{\mathrm{d}} \leq R_{\mathrm{d} 1} & 1^{\text {st }} \text { elastic branch } \\
R_{\mathrm{d}}=R_{\mathrm{d} 1}+K_{\mathrm{ed} 2}\left(D_{\mathrm{d}}-D_{\mathrm{d} 1}\right) & R_{\mathrm{d} 1} \leq R_{\mathrm{d}} \leq R_{\mathrm{d} 2} & 2^{\text {nd }} \text { elastic branch } \\
R_{\mathrm{d}}=R_{\mathrm{d} 2}+K_{\mathrm{ed} 3}\left(D_{\mathrm{d}}-D_{\mathrm{d} 2}\right) & R_{\mathrm{d} 2} \leq R_{\mathrm{d}} & 3^{\text {rd }} \text { elastic branch }
\end{array}
$$

where $D_{\mathrm{d}}, R_{\mathrm{d}}$ are the current values of displacement and force; $D_{\mathrm{d} 1}$ and $D_{\mathrm{d} 2}$ are the displacements corresponding to $R_{\mathrm{d} 1}$ and $R_{\mathrm{d} 2}$, Figure $7 \mathrm{~b}$. Analogous laws hold in the $\left(D_{\mathrm{d}} \leq 0, R_{\mathrm{d}} \leq\right.$ $0)$-region.

It is worth noting that each bumper relaxes to its original state after the impact and it may not be at rest when a new contact occurs. This depends on the ratio between the relaxation time of the bumper and the time interval between two subsequent impacts.

The comparison between experimental and numerical results, corresponding to the combination B4-G4-A3, was made with respect to the hysteresis loop of inertia force (Figure 8a) and the phase portrait (Figure $8 \mathrm{~b}$ ) in steady-state resonance condition. The solid lines represent the experimental results, and the dashed lines the numerical ones. The two vertical dashed lines identify the position of the bumpers. It can be observed that there is a good agreement between numerical and experimental results.

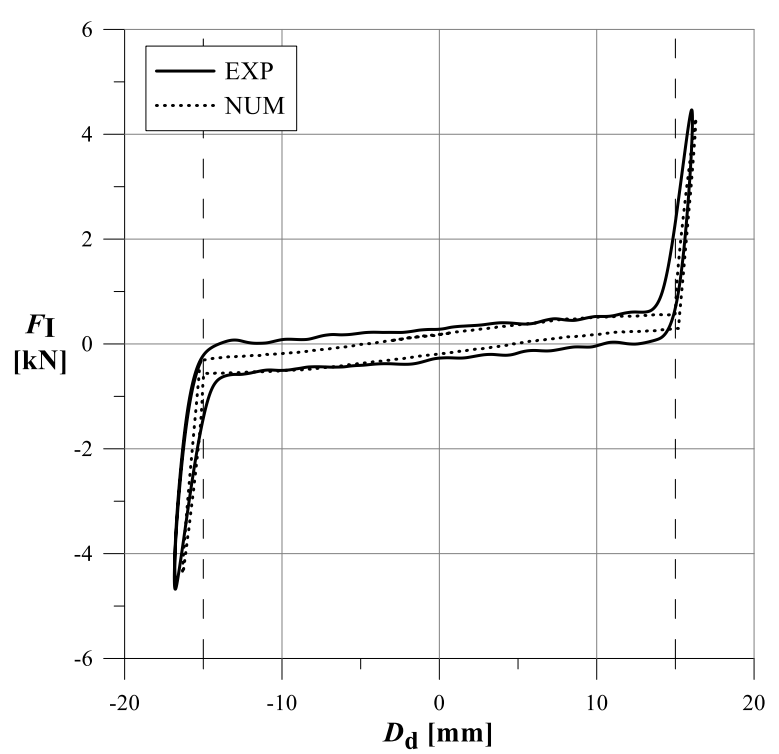

a)

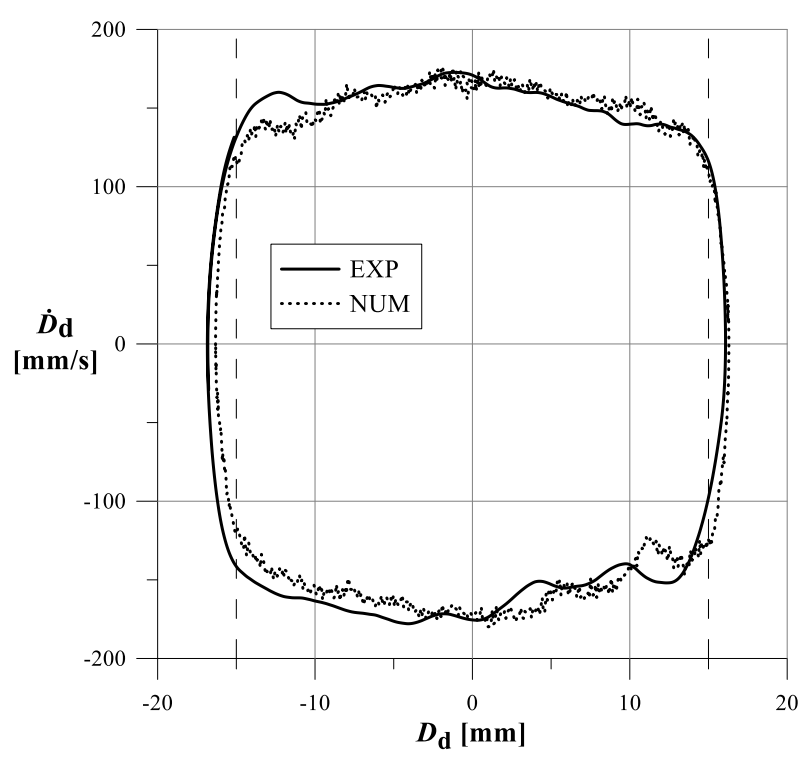

b)

Figure 8: Numerical vs. experimental results at resonance (B4-G4-A3): a) hysteresis loop; b) phase portrait. 


\section{CONCLUSIONS}

In this paper the experimental dynamic response of a base-isolated SDOF system, constrained by two symmetrically arranged deformable and dissipative bumpers and subjected to harmonic base excitation, is investigated. Three different peak values of table acceleration A, four amplitudes of the total gap $\mathrm{G}$ between mass and bumpers and four types of bumpers $\mathrm{B}$ were considered. The impact parameters (force and time of contact, coefficient of restitution and energy dissipated by the bumpers during the impact) were calculated from the experimental data. It was observed that they are influenced by the investigated parameters (A, G, B).

In particular, all the impact parameters, for almost all B-G pairs, increase with the peak table acceleration A. While bumpers B2 and B3, and in some cases also B4, behave in a similar way, the values of the parameters associated with B1 differ significantly.

Slightly different trends with the amplitude of the total gap $\mathrm{G}$ were observed; $\mathrm{G}$ appears to influence the impact parameters to a lesser extent, except for some combination B-A, that show quite different trends.

Finally, the more the bumper is rigid (from B1 to B2), the lower the penetration of the mass into the bumper. Consequently, both the contact time and the energy dissipated decrease, while the contact force increases. As concerns the coefficient of restitution, it shows an increase passing from B1 to B2 then it stands at a constant value or decreases.

The experimental results were used to identify the parameters of a numerical model that has been shown to reproduce in a sufficiently accurate manner the results obtained experimentally.

\section{REFERENCES}

[1] J.M. Kelly, Earthquake Resistant Design with Rubber. Springer, London, 1997.

[2] M. Ismail, J. Rodellar, F. Pozo, Passive and hybrid mitigation of potential near-fault inner pounding of a self-braking seismic isolator. Soil Dynamics and Earthquake Engineering, 69, 233-250, 2015.

[3] A. Reggio, M. De Angelis, Optimal design of an equipment isolation system with nonlinear hysteretic behavior. Earthquake Engineering and Structural Dynamics, 42, 1907 1930, 2013.

[4] A. Reggio, M. De Angelis, Combined primary-secondary system approach to the design of an equipment isolation system with High-Damping Rubber Bearings. Journal of Sound and Vibration, 333, 2386-2403, 2014.

[5] U. Andreaus, M. De Angelis, Nonlinear dynamic response of a base-excited SDOF oscillator with double-side unilateral constraints. Nonlinear Dynamics, 84, 1447-67, 2016.

[6] U. Andreaus, P. Baragatti, M. De Angelis, S. Perno, A Preliminary Experimental Study About Two-Sided Impacting SDOF Oscillator Under Harmonic Excitation. Journal of Computational and Nonlinear Dynamics, 12, 061010, 2017.

[7] U. Andreaus, P. Baragatti, M. De Angelis, S. Perno, Shaking table tests and numerical investigation of two-sided damping constraint for end-stop impact protection. Nonlinear Dynamics, 90, 2387-421, 2017. 
[8] K.H. Hunt, F.R.E. Crossley, Coefficient of restitution interpreted as damping in vibroimpact. Journal of Applied Mechanics, 42, 440-445, 1975.

[9] W. Goldsmith, Impact: The Theory and Physical Behaviour of Colliding Solids. Edward Arnold Ltd, London, 1960.

[10] R. Seifried, W. Schiehlem, P. Eberhard, Numerical and Experimental evaluation of the coefficient of restitution for repeated impacts. International Journal of Impact Engineering, 32, 508-524, 2005.

[11] U. Andreaus, P. Casini, Dynamics of SDOF Oscillators with Hysteretic MotionLimiting Stop. Nonlinear Dynamics, 22, 155-174, 2000.

[12] F. Naeim, J.M. Kelly, Design of Seismic Isolated Structures: From Theory to Practice. John Wiley and Sons, Chichester, U.K., 1999. 